\title{
RIPRAP STABILITY ON THE INNER SLOPES OF MEDIUM-HEIGHT BREAKWATERS
}

\author{
Henk Jan Verhagen ${ }^{1}$ \\ Bas van Dijk ${ }^{2}$ \\ Andries Nederpel ${ }^{2}$
}

\begin{abstract}
Medium height breakwaters are subjected to a considerable amount of overtopping. This implies the armour on the inner slope has to stable against overtopping waves. Existing equations do lack some basic understanding of the process of starting up movement of rock on the inner slopes.
\end{abstract}

In order to get more insight into this phenomenon a special device has been constructed in the laboratory. In this device it is possible to generate one single, overtopping wave. So with this piece of equipment overtopping of single waves can be repeated several times and a relation can be looked for between the individual plunge and the stability of the individual blocks. In an other research program the relation between the random waves and the individual plunges is investigated. As a follow up the results of damage by individual waves will be combined with the distribution of overtopping water over a breakwater. This combination will result in a damage distribution due to random overtopping.

It has been found from the first test series that stability of the blocks can be described as a function of the maximum water velocity on the crest, combined with some geometrical parameters of the inner slope. From this research followed that the crest height and inner slope also had an effect on stability.

\section{Introduction}

Medium height breakwaters are subjected to a considerable amount of overtopping. This implies the armour on the inner slope has to stable against overtopping waves. Literature reveals the existence of a few formula to calculate the required block size, but all of them are related to the wave height on the outer side of the breakwater (e.g.

1 Ass. professor in coastal engineering, Delft University of Technology, P.O. Box 5048, NL2600DA Delft, The Netherlands, H.J.Verhagen@ct.tudelft.nl

2 M.Sc. student, Delft University of Technology 
WALKeR, ET.AL [1976], VidAL ET.AL.[1990] and KoBAYASHI AND KudALE[1996]). However, these equations do lack some basic understanding of the process of starting up movement of rock on the inner slopes.

In case one relates the stability of the blocks on the inner slope directly to the waves approaching the breakwater, the model should include not only the wave parameters (e.g. wave height, period, spectral shape, etc.) but also a number of the parameters of the construction, like the roughness, the breakwater height, the permeability, the outer and the inner slope, and many more. When performing such tests, it is not possible to separate the effect of all individual parameters on the stability, unless a very huge number of tests are carried out.

Therefore, the idea is to separate the problem into two parts:

- the incoming wave gives an amount of overtopping over and infiltration into the breakwater

- the overtopping and the outflow cause an instability of the blocks on the inner slope.

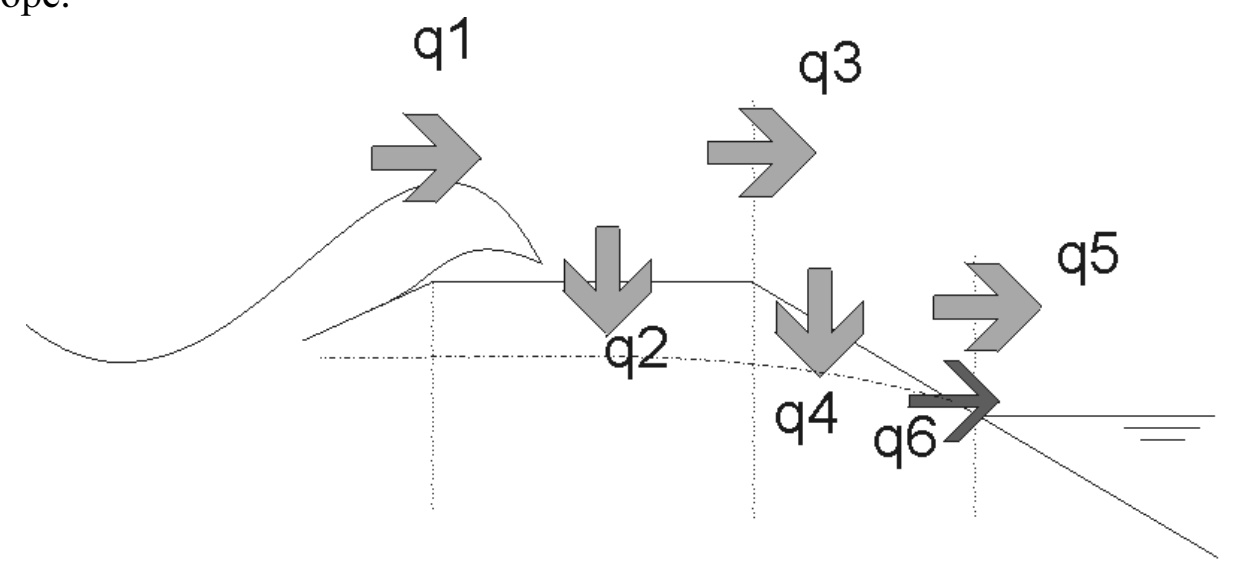

Figure 1: distribution of the overtopping quantities

In separate research the quantities $\mathrm{q} 1, \mathrm{q} 2$ and $\mathrm{q} 3$ are determined (see figure 1). This research focussed on the effect of q5 on the stability of the armour in the inner slope of the breakwater. For the time being, the effect of $\mathrm{q} 6$ is not considered. However it is envisaged that q6 may have an additional effect on the stability of the blocks. This will be subject to future research.

In order to get more insight into the effect of the overtopping quantity q5, a special device has been constructed in the laboratory. In this device it is possible to generate one single, overtopping wave. So with this piece of equipment overtopping of single waves can be repeated several times and a relation can be looked for between the individual plunge and the stability of the individual blocks. 


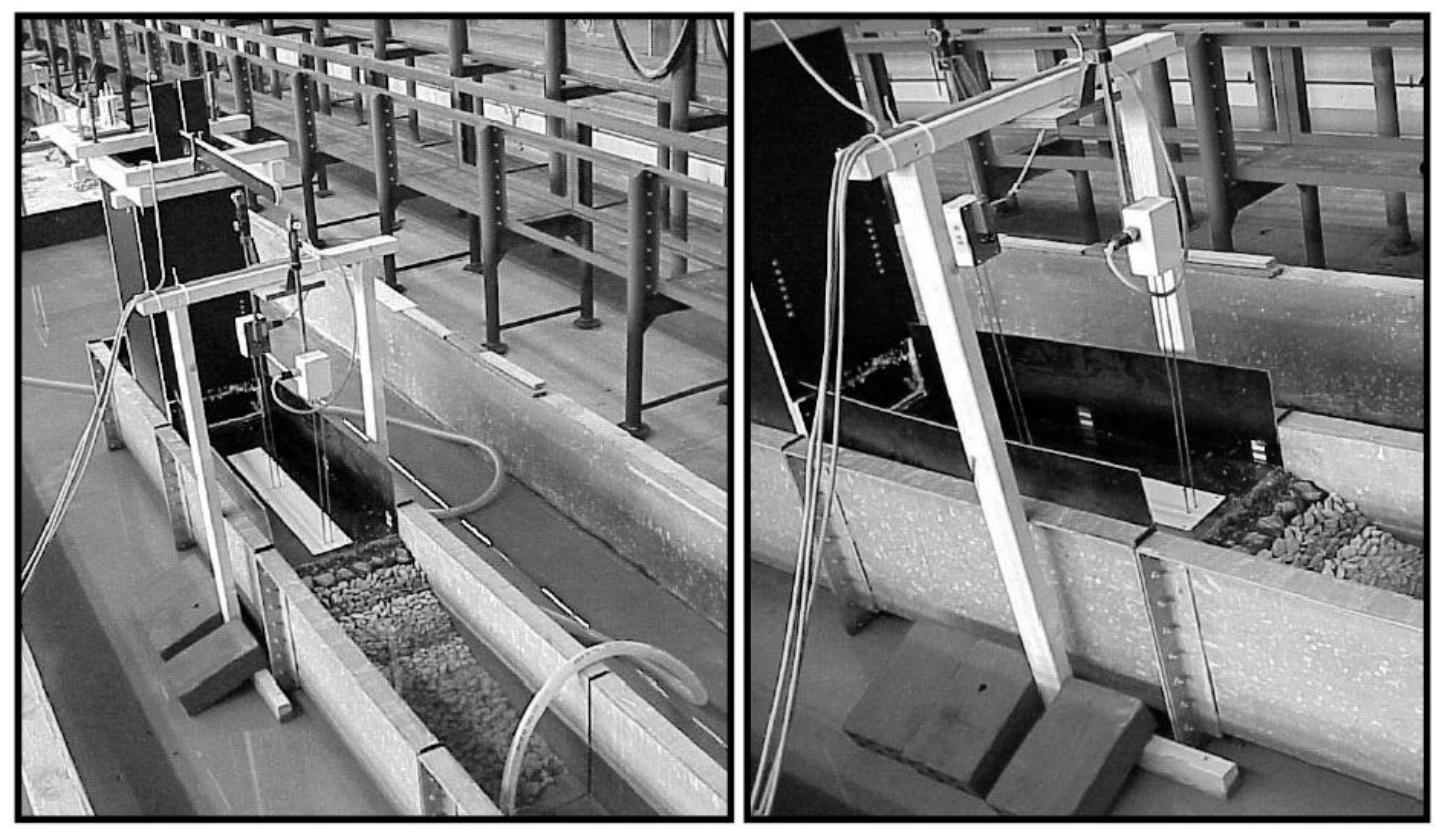

Figure 2: the wave impact device in the laboratory

Overtopping waves ('plunges') were simulated with water flowing out of a reservoir. The breakwater model was reduced to the rear slope armour layer only. In this way the amount of influencing parameters was minimised. Implicitly, this leads to a simple laboratory model instead of a complicated scale model of a prototype of reality.

In the experiments the rear slope was subjected to plunges of which the sizes were increased with certain steps by increasing the water volume in the reservoir. One step consisted of three plunges of the same size. After each plunge the number and location of the displaced stones were recorded. The experiment ended when rear slope collapse occurred.

From the experiments it was concluded that a distinction has to be made between the occurrence of damage and the collapse behaviour of the rear slope. First, the occurrence of damage is considered. As long as the rear slope is not collapsed, the damage can be related to the quotient of the maximum instantaneous discharge of the plunge and the maximum layer thickness of the plunge. This parameter is defined as the characteristic velocity of the plunge. The volume of the plunge does not influence the rear slope stability.

It is found that the stability can be described in an optimal way using as stability parameter:

$$
\Theta_{u_{\text {char }}}=\frac{u_{\text {char }}^{2}}{\Delta g D_{n 50}}
$$


In this equation the characteristic velocity $u_{\text {char }}$ is the time averaged velocity during the plunge of the wave (in fact it is the quotient of the maximum instantaneous discharge and the maximum layer thickness of the wave.), $\Delta$ is the relative density of the rock and $D_{n 50}$ is the nominal median diameter of the rock.

The damage to a slope is expressed in the damage number $N_{o d}$, defined as:

$$
N_{o d}=\frac{i}{B / D_{n 50}}
$$

in which $B$ is the width of the flume and $i$ the number of displaced stones in the experiment.

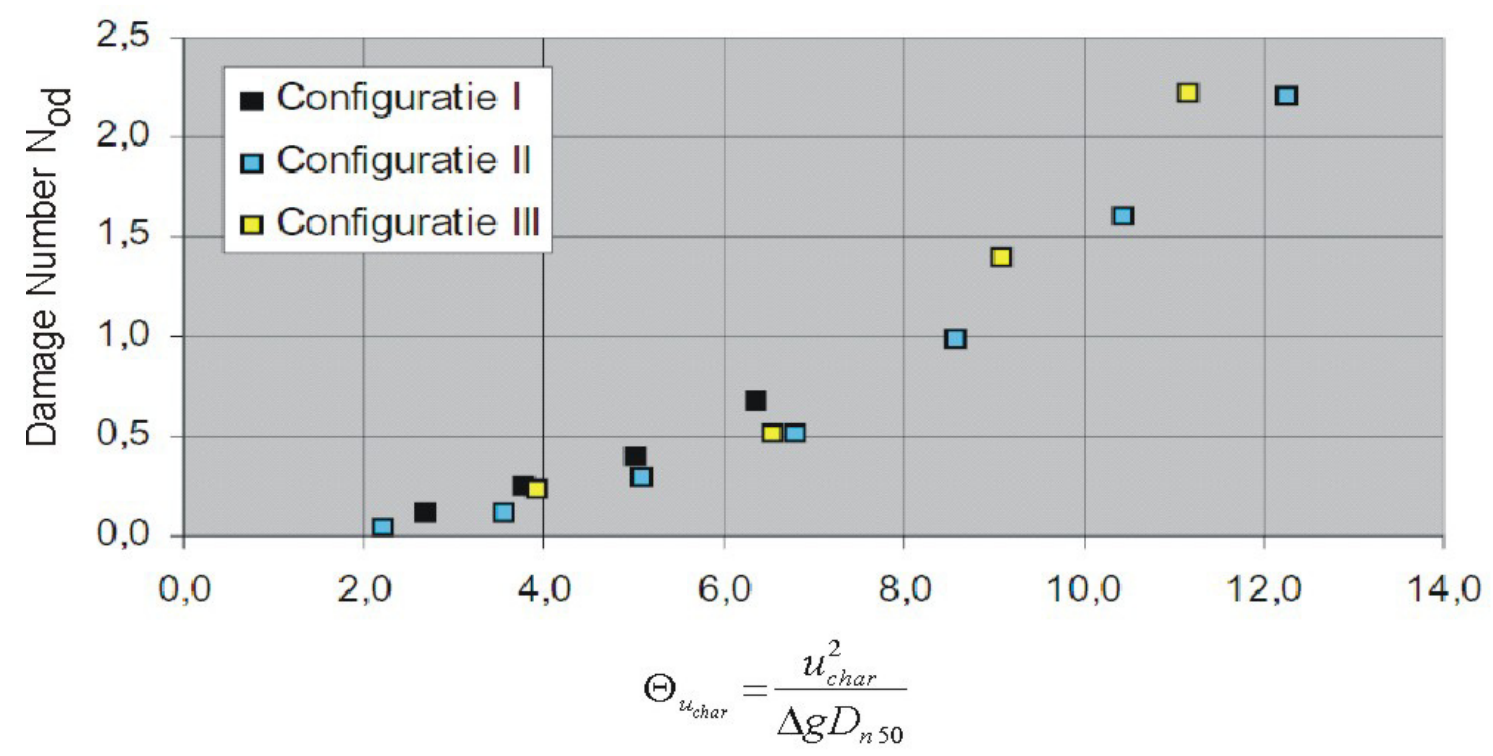

Figure 3: damage as function of the characteristic velocity

The damage increases progressively, with increasing characteristic velocity. The spread in damage between repeated experiments also increases with increasing characteristic velocity. The damage mostly occurred just below the waterline and due to the loss of support consequently also above the waterline. Under water the damage quickly decreases. For a given value of the stability parameter $\Theta$ the damage number $\mathrm{N}_{\text {od }}$ can be determined easily if there was no failure in any of the experiments. However, if in some of the experiments the inner slope has failed, one cannot determine the average value any more, and it is not possible to determine $\mathrm{N}_{\text {od }}$. For values of $\Theta<8.6$ there has been no failure.

It was found that the probability of failure was higher in case of larger damage, however it is not possible to state that in case damage is more than a given value, failure will always occur. 


\section{Freeboard height and number of waves}

The crest freeboard significantly influences the rear slope damage development. Similar series of experiments were carried out for different crest freeboards by varying the water level in the flume. The rear slope stability is least for an intermediate crest freeboard. On average, the sum of the stabilising damping effect of the tailwater and the destabilising lifting effect of the tailwater is least favourable in this situation. According to the experiments, the crest freeboard seems to influence the collapse behaviour. Especially in the case of a very low crest freeboard, the rear slope seems less sensitive to collapse than in the case of a high crest freeboard or an intermediate one.

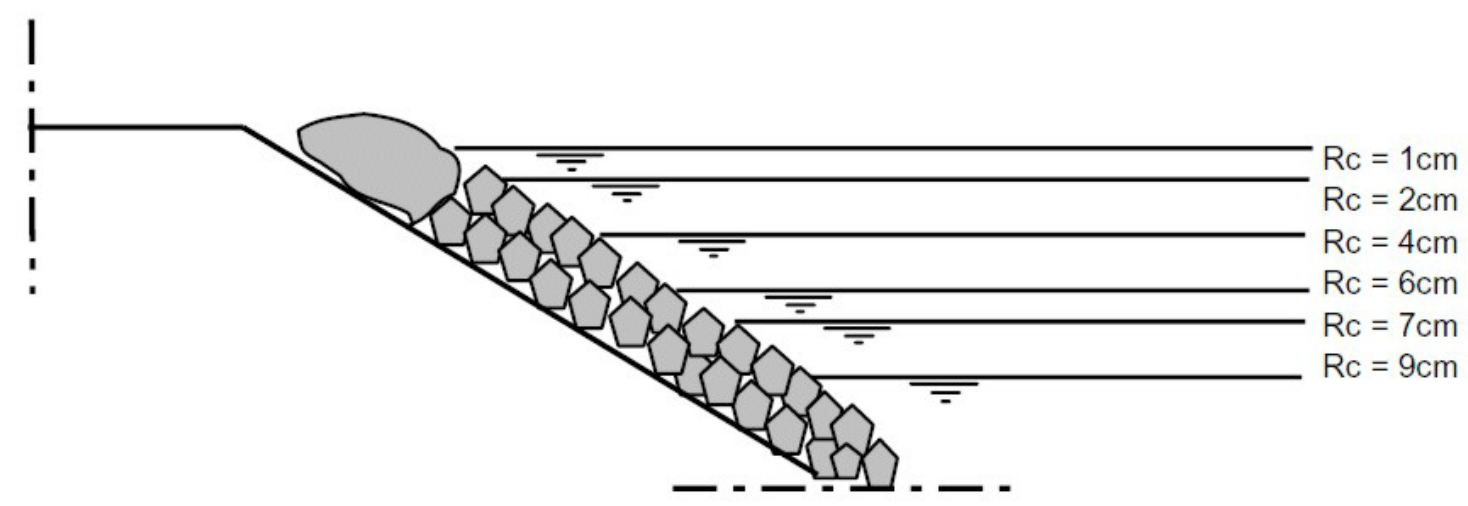

Figure 4: several levels of freeboard

Six different crest heights are investigated for a slope angle of 1:1.5. Considerable damage occurs for a crest high above the waterline. Only few waves are necessary to accomplish this damage. As the crest height diminishes, the damage diminishes for the same amount of waves. A relation between crest height and stability seems obvious. The damage development can be related to a combination of characteristic velocity and crest height.

In every step, consisting of three waves, the first wave causes most damage. After the following two waves, hardly additional damage occurs. The armour stone seems to restructure and will be more stable for this wave size. For the breakwater with the lower crest, the next step to an increased wave size will be relatively smaller compared to the breakwater with higher crest. More waves are needed to cause the same damage for the lower crest. Because of the smaller relative step size, the breakwater with a lower crest is more stable due to a better restructuring of the armour stone. Experiments were carried out with a smaller step size and were compared to experiments with a normal step size. The damage development seems to resemble when the square root of the number of waves is added to the relation between damage development and stability. In order to include this in the stability parameter, the stability parameter has been extended to: 
$\Theta_{u_{c h a r}, R_{c}, i}=\frac{u_{c h a r}^{2}}{\Delta g D_{n 50}} \frac{R_{c}}{D_{n 50}} \sqrt{i}$

in which $R_{c}$ is the freeboard (crest height above water) and $i$ the number of waves in the experiment.

However there are a number of additional conclusions from the tests:

- Above the waterline there is always large damage, due to both the effect of the direct impact of the wave on the stones above the waterline, as well as loss of support because of movement of stones in a lower position.

- Most damage occurs at the location of the waterline itself. Stones are here fully subjected to the impact force of the wave, but have less stability because of the buoyancy. Stones just below the waterline suffer less from the impact, because the damping effect of the still water. Stones at the waterline will move first, and consequently deprive the higher placed stones from their support.

- Below the waterline the damage decreases rapidly. Only damage is observed to the first 4 to 6 stones below the waterline, no significant damage could be observed to deeper lying stones.

\section{Effect of the slope}

The influence of slope angle can only be examined when the damage above the waterline for both slopes will be analysed. When using a gentler slope, it appears that less damage occurs for a certain value of $u_{c h a r, R c, i}$. By adding the influence of the angle in this relation, the damage development is still slightly more progressive for the steeper slope. However, there could be a relation with the angle of wave attack. The characteristic velocity parallel to the slope angle is less for the gentler slope.

Experiments were carried out for a more flat slope, 1:3, with three different crests heights. By examining more crest heights using this slope angle, the relation between stability and damage development, which is mentioned above, can be verified. Possibly more parameters can be of influence when investigating the damage development with a flatter slope. However, the damage area is larger. The complete development is not taken into consideration. For a better analysis the damage above the waterline is considered. The same relations for velocity, crest height and number of waves appeared.

The angle of wave attack compared to the slope angle has to be added to the relation for stability. By changing the slope angle, two influences for damage development are important: a hydraulic and a structural influence.

$\Theta_{u_{\text {char }}, R_{c}, i, \alpha}=\frac{u_{\text {char }}^{2}}{\Delta g D_{n 50}} \frac{R_{c}}{D_{n 50}} \sin (\alpha) \sqrt{i}$

in which $\alpha$ is the inner slope of the structure. 


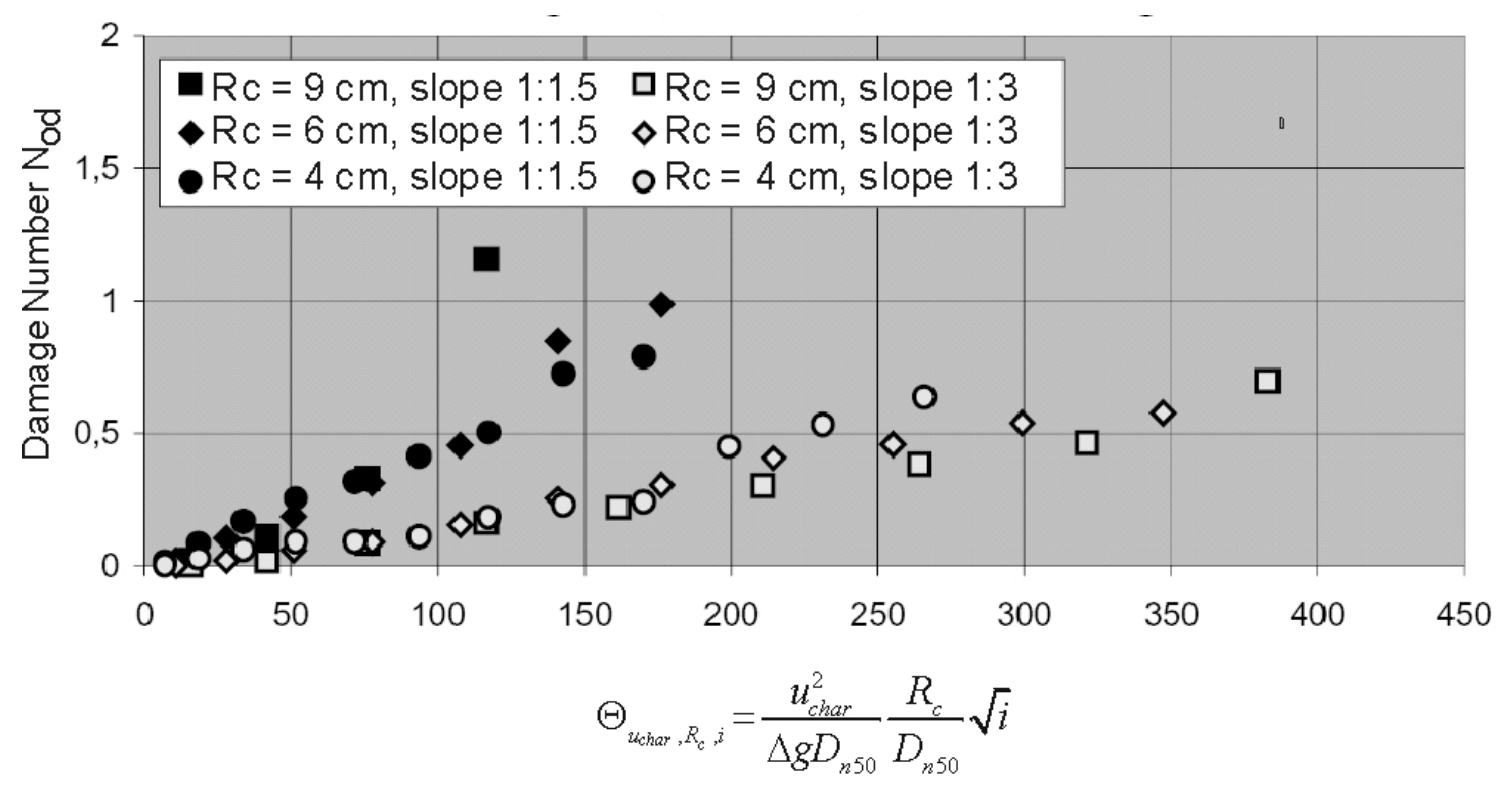

Figure 5: effect of the slope on stability

It can be concluded that the development of damage is related to a characteristic velocity parallel to the slope angle, the height of the crest, the number of waves and the angle of the slope. However, this conclusion should be handled with care, because of the large spread in results.

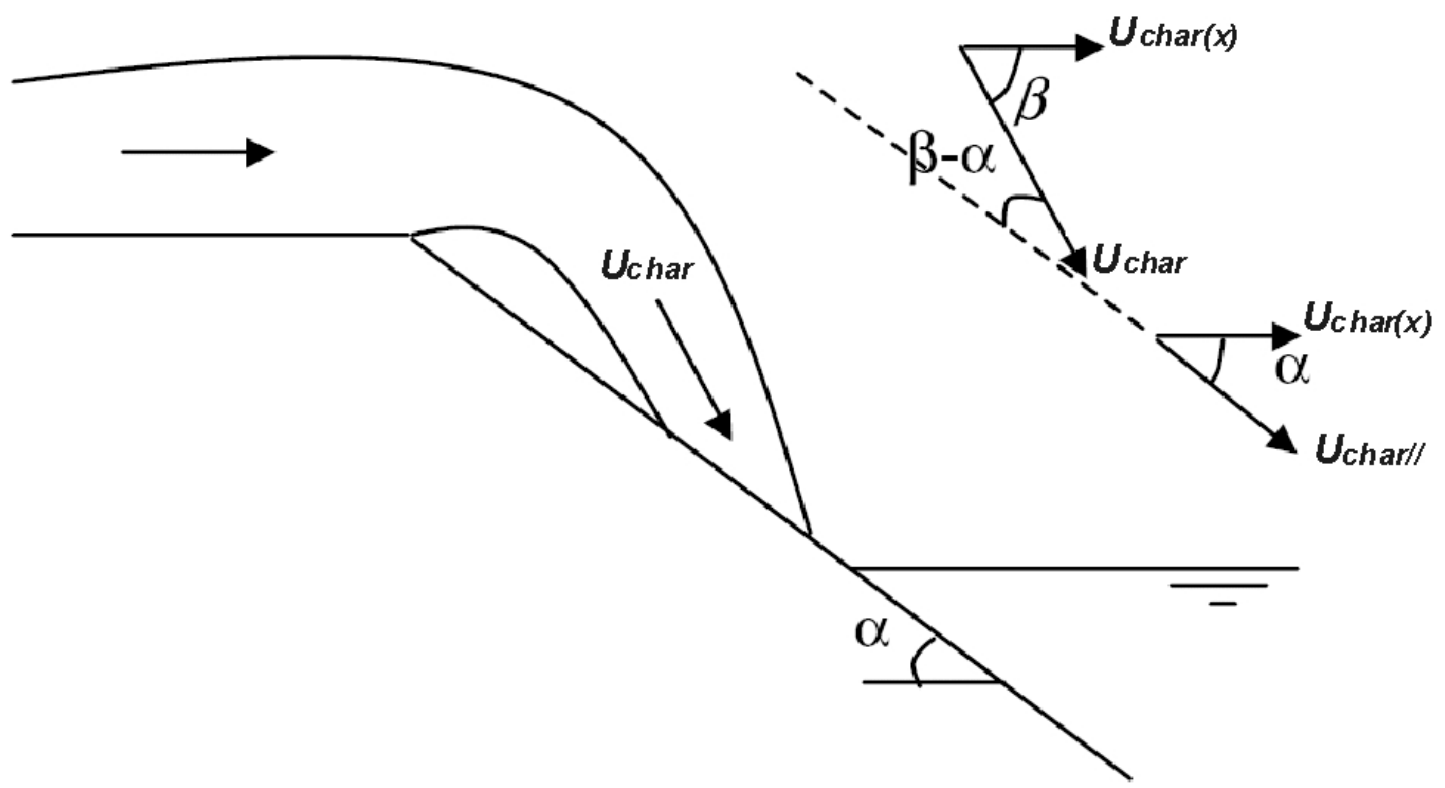

Figure 6: impact on a slope

More experiments are needed to confirm this conclusion. Moreover, only the damage above the waterline is considered. Influence of depth can also play an important role in rear slope stability. This parallel characteristic velocity can be expressed as: 
$u_{\text {char } / /}=u_{\text {char }} \cos (\beta-\alpha)$

in which $\beta$ is the approach angle of the wave on the slope. See also figure 6 .

Consequently, the stability parameter becomes:

$\Theta_{u_{\text {char }} / / R_{c}, \alpha, i}=\frac{\left(u_{\text {char }} \cos (\beta-\alpha)\right)^{2}}{\Delta g D_{n 50}} \frac{R_{c}}{D_{n 50}} \sin (\alpha) \sqrt{i}$

All data are plotted in figure 7 as a function of this damage number. Apart from the high freeboard values, it is clear that this parameter is able to predict the damage number $\mathrm{N}_{\mathrm{od}}$ quite accurately.

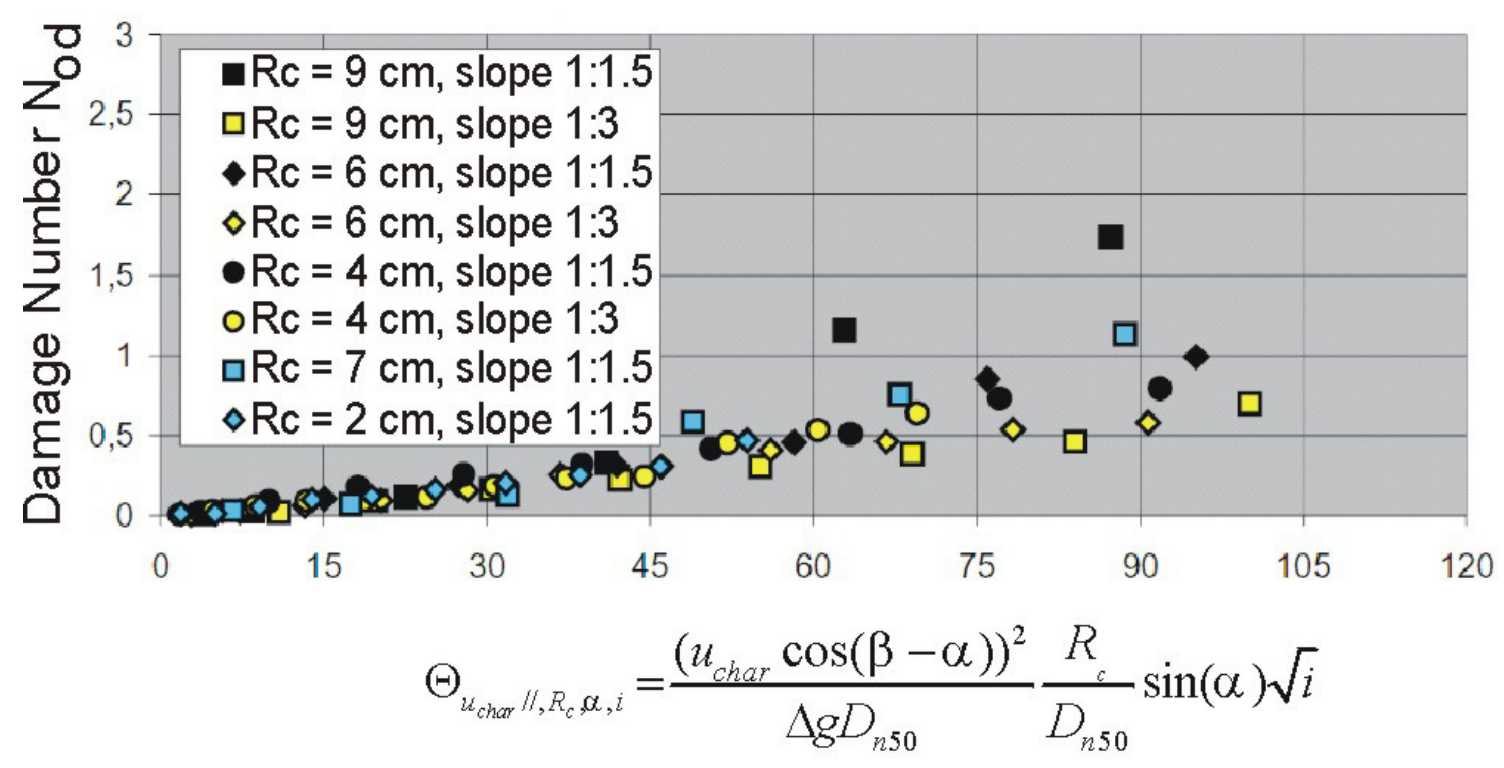

Figure 7: stability of an inner slope

\section{Damage by a single wave}

A few experiments were carried out for damage due to one certain wave. Two different crest heights were considered. An even larger spread in damage appeared for these experiments compared to the experiments with cumulative damage development. The larger spread is inherent to the experiment procedure. After one wave the slope was being rebuilt by randomly placed stones. As a result of examining the crest heights, the relation between velocity and crest height could only be checked. However, due to the large spread and the limited number of experiments, the uncertainty is too large to derive the same relation.

\section{Collapse behaviour}

Besides damage development, collapse behaviour can be considered. In some experiments, the rear slope shows collapses for relative small values of wave size, while in other experiments the rear slope will be able to resist considerable large values of the 
size of the wave, before collapse occurs. The probability of collapse increases, while increasing wave size. From results, it can be concluded that this probability is best characterised as a normal distribution. From this normal distribution, it can be noticed that the parameter for stability is also valid for the collapse behaviour regarding the step size and the slope angle. However, the probability of collapse is larger for breakwaters with a lower crest.

Results are plotted in figure 8, on the horizontal axis the stability number is given while on the vertical axis the probability of failure is given, computed as

$$
P_{\text {failure }}=\frac{n_{\text {failed }}}{n+1}
$$

in which $n_{\text {failed }}$ is the number of failed rear slopes and $n$ is the total number of experiments.

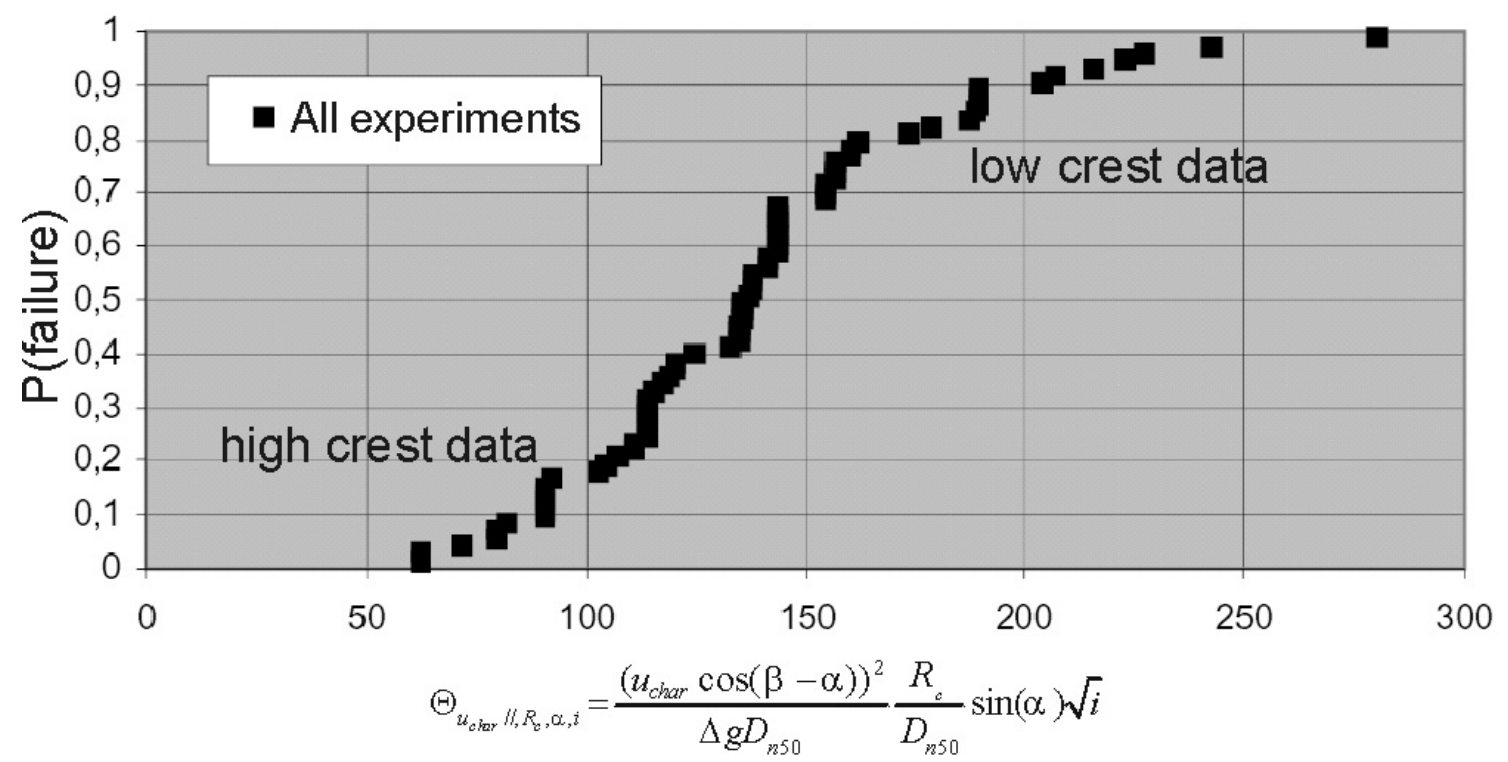

Figure 8: probability of failure as function of stability number

This implies the parameter for stability is not valid regarding the crest height. The collapse behaviour would be randomly divided on the normal distribution. In future research it, it is recommended to make a distinction between damage development and collapse behaviour of a breakwater. Experiments have to be repeated many times in order to achieve more reliability in damage development and collapse behaviour. Both influence of wave velocity and direction of wave attack need more attention in order to better investigate collapse behaviour and damage development. In addition, the influence of number of waves has to be investigated more thoroughly. Furthermore, future research has to focus upon more structural parameters like average stone size and stone shape. The field of interest has to be large enough in order to investigate the influence of waterdepth. Finally, examining the stability of one single stone will lead to a better understanding of the rear slope physics. 
It was found that two separated phenomena had to be considered, the gradual damage of the slope and the collapse of a large part of the inner slope. The probability of collapse increases when the amount of gradual damage has increased. But because of the wide standard deviation in both processes, one cannot combine them to one process in the computation. Also was found that the ratio between inner water level, crest height and impact height of the plunge has a large influence on the amount of local damage.

\section{Conclusions}

- An experimental relation has been derived between speed of overtopping water and stability of individual rock elements on the inner slope of a breakwater.

- This relation depends on the number of waves, and is therefore difficult to use in case of random waves.

- The effect of the freeboard is complicated. The parameter "dimensionless freeboard" $\left(\mathrm{R}_{\mathrm{C}} / \mathrm{D} 50\right)$ does not really describe the process completely.

\section{Acknowledgments}

The tests were all done in the Fluid Mechanics Laboratory of Delft University of Technology; tests were funded from the general research budget of the Section of Hydraulic Engineering.

\section{References}

Kobayashi, N, Kudale, M.D.,[1996] "Hydraulic stability analysis of lee-side slopes of overtopped breakwaters", Proceedings ICCE

NEDERPEL, A [2002] Stabiliteit van het achtertalud van een breakwater. M.Sc.-thesis, Delft University of Technology, $112 \mathrm{pp}$

VAN DIJK, B. [2001] The rear slope stability of rubble mound breakwaters. M.Sc.thesis Delft University of Technology, $154 \mathrm{pp}$

Vidal, C., Losada, M.A., Medina, R., Mansard, R., Gomez-Pina, G. [1990] A Universal Analysis for the Stability of both Low-crested and Submerged Breakwaters, Proceedings ICCE

Walker, J.R., PALmer, R.Q. AND Dunham, J.W, [1976] Breakwater slope stability, proc. Civ. Eng in Oceans 